\title{
Calculation of the Duty Cycle for BECA
}

\author{
Chiranjib Patra \\ Calcutta Institute of Engineering and Mangement, \\ Kolkata
}

\author{
Sourish Mullick \\ Calcutta Institute of Engineering and Mangement, \\ Kolkata
}

\begin{abstract}
Consumption of energy is one of the major problems in wireless sensor network, as sensor nodes driven on battery power. Therefore, it is intended to minimize the power consumption by putting the wireless interface of the sensor nodes in low power sleep mode. Wireless sensor networks constructed with hundreds and thousands of wireless sensor nodes and they deployed to solve different problems of our life. Therefore, design solutions for reduced resource consumption of wireless nodes have a remarkable impact in green network setup. Probability of each state has to be determined to analyze the energy consumption in duty cycle. In this paper steady state probability of sensor nodes are analyzed and derived, i.e., sleep, listen, and active states, in terms of traffic characteristics and timer values, i.e., sleep timer, listen timer, and active timer.
\end{abstract}

This work can be a guideline for appropriate timer selection for sensor network can be achieved for efficient energy conservation and the methodology developed here can lead to other duty cycle based energy conservation schemes with different sensor nodes.

\section{General Terms}

Sensor network; energy conservation; duty cycling; state probability

\section{Keywords}

Sensor Network; Energy Conservation; Duty Cycling; Steady; Semi-Markov Process Approach; State Probability

\section{INTRODUCTION}

Multiple sensor nodes and a sink node form a sensor network. These sensor nodes are used to monitor and measure traffic or public movement, temperature, humidity, pressure, etc. [1,2] A sensor sensed information is transmitted to a sink node through multi-hop routing and finally reaches to the central management system. The deployed sensor nodes may have to remain for few years as well and in real world scenarios, it's impossible to change the battery of those sensors once they are deployed. Therefore, to get a certain level of efficiency energy conservation or power control is one of the most critical issues in sensor network [3].

A well known fact is that the energy required for communication is much higher than that due to either sensing information or processing sensed information [4, 5]. Consumption of energy for communication, just listening communication interface also consumes comparable power to receiving data and the ratio of energy consumption among listening, receiving, and transmitting data respectively is about 1,1 , and 1.5 . [5, 6].In order to save energy it is important to operate the communication interface of sensor nodes in low power sleep state when there is no communication required with its neighbor. [7] To increase the lifetime of a sensor node, many researchers have been done and many algorithm has been proposed mostly on duty cycle, data-driven approaches, and mobility [4].By putting unused sensor nodes in low power sleep mode the duty cycling is achieved. Data-driven approaches make the power management by reducing the number of sampled data by eliminating redundant data delivery to the sink node by using correlation property between sampled data [8]. Limited multihop routing is another way of reducing power consumption for mobile sensor nodes. In this paper we are focused mainly on duty cycling technique to reduce power consumption as this scheme is considered the most suitable power conservation technique [4]. In topology control, sensor nodes are used redundantly and for network connectivity a set of sensors are in active mode, and the other sensor nodes are deactivated for power saving. Thus, it is important to identify the activated or deactivated sensors in topology control. Different levels of power consumption, such as sleep, listen and power management for efficient power conservation controls active states. Initially nodes remains in sleep state when communication is not required, by putting communication interface in the low power sleep state. Nodes periodically wake up from the sleep state to listen state and listens to the radio interface. In listen state, if it doesn't senses any data intended for it, it moves back to sleep state. Otherwise, its state changes to active state and communication took place with other sensor nodes. In active state in case of no further data to be transmitted or received, it waits until the active timer expires, after that, it moves to sleep state again.

Different states of sensor nodes have difference in their power consumption, so in order to analyze the consumption of energy it is required to obtain the steady state probability of sensor nodes [3].

In our work we analyze steady state probability using Markov's chain to deduce the effect of the assumption of considering a non event state and event state. This assumption leads us to an alternative understanding of sleep cycle management, which may be helpful in calculating the maximum energy consumed by the sensor in a cycle and hence minimize upon the energy function.

\section{RELATED WORK}

In this section, there is a survey on related works on energy conservation schemes based on duty cycling. As mentioned in Introduction, topology control and power management are the classification of duty cycling. In addition, activation and deactivation of sensor nodes by topology control is further divided into location driven protocol and connectivity driven protocol. [4]. in location driven protocol, the criteria is the location of sensor nodes and geographical adaptive fidelity (GAF) [8] is a representative example of this protocol. In connectivity driven protocol, the criteria is network connectivity and Span [7] is one example of this protocol.

In GAF [8], sensor network is divided into grids, here the nodes in adjacent grid can communicate and only one member 
in a grid remains in active state others remain in sleep state. There are three states in GAF, i.e., sleep, discovery, and active states. A node in sleep state remains in the same state until the expiration of sleep timer. After expiration of sleep timer it moves in discovery state. The sensor node exchanges discovery messages with other sensor nodes within the same grid, in discovery state. If higher ranked node i.e., higher residual energy, sends a discovery message to a sensor node within discovery timer, received node moves back to sleep state. Otherwise, it moves to active state. In active state, a discovery message from a sensor node with higher rank within active timer, makes it to move to sleep state. Otherwise, it moves back to discovery state at the expiration of active timer.

Span [7] is a distributed backbone selection protocol where sensor network coodinator is elected. The coordinators are responsible for multi-hop routing while other sensor nodes are sleeping. Sensor nodes in sleep state periodically wake up to check whether to sleep or stay awake as a coordinator. Coordinator selected by eligibility rule, which is based on the battery level and the number of neighbor sensor nodes. In coordinator eligibility rule, if two neighbor sensors became unreachable from each other then the non-coordinator sensor node becomes a coordinator sensor node. Each coordinator periodically checks the reachability of each pair of sensor nodes to withdraw a coordinator if it can be removed without hampering the communication.

Basic energy conservation algorithm (BECA) [5], if there is no requirement of communication a sensor nodes stay in sleep state, by putting the communication interface in the low power sleep state. Each sensor node periodically wakes up for after every sleep timer, Ts, each sensor wakes up by moving into listen state to sense any incoming data to the sensor node. During listen timer, $\mathrm{Tl}$, if there is no incoming data until the expiration of the listen timer, it moves back to sleep state again. Otherwise, its state changes to active state and it communicates with another sensor node. In active state, once transmit or receive of data is over it waits until the expiration of active timer, Ta, and it moves to sleep state again.

The rest of our article is organized as follows section 2 deals with Modeling and analysis of sensor node transition model, section 3 details out for the evaluation of duty cycle, section 4 exemplifies our equation into evaluation of real life protocol with parameters related to energy, transitional probability and network constants.

\section{MODELING AND ANALYSIS OF SENSOR NODE STATE TRANSITION MODEL}

For mathematical derivation [9], In this protocol we have the event driven scenario where each sensor node follows a possion process. This assumption is justified as the events like sleep, wake, forward, transmit are independent of each other. This assumption is helpful in understanding the mechanics of the analytical model into consideration. The analytical model is helpful in calculating the probabilities of the sensor node states. The above-described model can be used to apply in other traffic models with usual modifications. Now considering the above discussions of the model we shall deduce the probability of every state of occurrence

Let the transmitting, receiving, and forwarding data packets at a sensor node occur according to a Poisson process with parameters $\lambda \mathrm{t}, \lambda \mathrm{r}$, and $\lambda \mathrm{f}$, respectively;
The time duration that a sensor node remains in activetransmit, active-receive, and active-forward states follows an exponential distribution with a mean value of $1 / \mu \mathrm{t}, 1 / \mu \mathrm{r}$, and $1 / \mu \mathrm{f}$

The values of sleep timer, listen timer, and active timer are assumed as constant and they are denoted by $\mathrm{Ts}, \mathrm{Tl}$, and $\mathrm{Ta}$, respectively

We denote sleep, listen, active-transmit, active-receive, active-forward, and active-idle states as states 1, 2, 3, 4, 5, and 6 , respectively, for notational convenience. Since the residence times of a sensor node in sleep, listen, and activeidle states do not follow an exponential distribution, the sensor node state transition behavior is analyzed using a semiMarkov process approach

The steady state probability of each sensor node state can be obtained as

$$
P_{k}=\frac{\pi_{k} \overline{t_{k}}}{\sum_{i=1}^{6} \pi_{i} \bar{t}_{i}}
$$

Where $\pi \mathrm{k}$ denotes the stationary probability of state $\mathrm{k}$ and tk is the mean residence time of the sensor node in state $\mathrm{k}$. The stationary probability is obtained by solving the following balancing equations

$$
\begin{aligned}
& \pi_{j}=\sum_{k=1}^{6} \pi_{k} P_{k j} \quad j=1,2,3, \ldots .6 \\
& 1=\sum_{k=1}^{6} \pi_{k}
\end{aligned}
$$

where Pkj represents the state transition probability from state $\mathrm{k}$ to state $\mathrm{j}$. The state transition probability matrix $\mathrm{P}=[\mathrm{pkj}]$ of the state transition model is given by

Where

$\mathrm{P}_{12}=$ sleep-listen

$\mathrm{P}_{13}=$ sleep-active transmit

$\mathrm{P}_{21}=$ listen-sleep

$\mathrm{P}_{23}=$ listen -active transmit

$\mathrm{P}_{24}=$ listen -active receive

$\mathrm{P}_{25}=$ listen-active forward

$\mathrm{P}_{36}=$ active transmit-active idle

$\mathrm{P}_{46}=$ active receive-active idle

$\mathrm{P}_{56}=$ active forward -active idle

$\mathrm{P}_{61}=$ active idle-sleep

$\mathrm{P}_{63}=$ active idle-active transmit

$\mathrm{P}_{64}=$ active idle-active receive 
$\mathrm{P}_{65}$ active idle-active forward

State transition probability $\mathrm{P}_{\mathrm{kj}}$ can be derived based on the distribution of time states $\mathrm{k}$ to $\mathrm{j}, \mathrm{T}_{\mathrm{kj}}$

Exit from the sleep state is caused by any of the following events:

Sleep timer expiration, a transmitting packet interval $T_{13}$

Then the state transition probabilities are obtained as [9]

$P_{12}=e^{-T_{s} \lambda_{t}}$

$P_{13}=1-P_{12}$

$P_{21}=e^{-\left(\lambda_{t}+\lambda_{r}+\lambda_{f}\right) \mathrm{T}_{t}}$

$P_{23}=\frac{\lambda_{t}}{\lambda_{t}+\lambda_{r}+\lambda_{f}}\left(1-\mathrm{e}^{-\left(\lambda_{t}+\lambda_{r}+\lambda_{f}\right) \mathrm{T}_{l}}\right)$

$P_{24}=\frac{\lambda_{r}}{\lambda_{t}+\lambda_{r}+\lambda_{f}}\left(1-\mathrm{e}^{-\left(\lambda_{t}+\lambda_{r}+\lambda_{f}\right) \mathrm{T}_{l}}\right)$

$P_{25}=\frac{\lambda_{f}}{\lambda_{t}+\lambda_{r}+\lambda_{f}}\left(1-\mathrm{e}^{-\left(\lambda_{t}+\lambda_{r}+\lambda_{f}\right) \mathrm{T}_{l}}\right)$

$P_{61}=e^{-\left(\lambda_{t}+\lambda_{r}+\lambda_{f}\right) \mathrm{T}_{a}}$

$P_{63}=\frac{\lambda_{t}}{\lambda_{t}+\lambda_{r}+\lambda_{f}}\left(1-\mathrm{e}^{-\left(\lambda_{t}+\lambda_{r}+\lambda_{f}\right) \mathrm{T}_{a}}\right)$

$P_{64}=\frac{\lambda_{r}}{\lambda_{t}+\lambda_{r}+\lambda_{f}}\left(1-\mathrm{e}^{-\left(\lambda_{t}+\lambda_{r}+\lambda_{f}\right) \mathrm{T}_{a}}\right)$

$P_{65}=\frac{\lambda_{f}}{\lambda_{t}+\lambda_{r}+\lambda_{f}}\left(1-\mathrm{e}^{-\left(\lambda_{t}+\lambda_{r}+\lambda_{f}\right) \mathrm{T}_{a}}\right)$

Similarly we can calculate the residence time for each transition probability[9]

$\mathrm{T}_{13}=\frac{1-e^{-\lambda_{t} T_{s}}}{\lambda_{t}}$

$\mathrm{T}_{12}=\mathrm{T}_{1}$

$\mathrm{T}_{21}=\mathrm{T}_{1}$

$\mathrm{T}_{23}=\frac{1-e^{-\left(\Lambda T_{l}\right)}}{\Lambda}$

$\mathrm{T}_{24}=\frac{1-e^{-\left(\Lambda T_{l}\right)}}{\Lambda}$
$\mathrm{T}_{25}=\frac{1-e^{-\left(\Lambda T_{l}\right)}}{\Lambda}$

$\mathrm{T}_{36}=\frac{1}{\mu_{t}}$

$\mathrm{T}_{46}=\frac{1}{\mu_{r}}$

$\mathrm{T}_{56}=\frac{1}{\mu_{f}}$

$\mathrm{T}_{61}=\mathrm{T}_{\mathrm{s}}$

$\mathrm{T}_{63}==\frac{1-e^{-\left(\Lambda T_{a}\right)}}{\Lambda}$

Similarly we have

$\mathrm{T}_{64}=\frac{1-e^{-\left(\Lambda T_{a}\right)}}{\Lambda}$

$\mathrm{T}_{65}=\frac{1-e^{-\left(\Lambda T_{a}\right)}}{\Lambda}$

Now considering the Markov chain for the node considered we describe Markov transition probability matrices when there is event and when there is no event. The diagrams below depict the non event and event cases.

These are the possible allowed states.

Now state diagram for NON-EVENT:

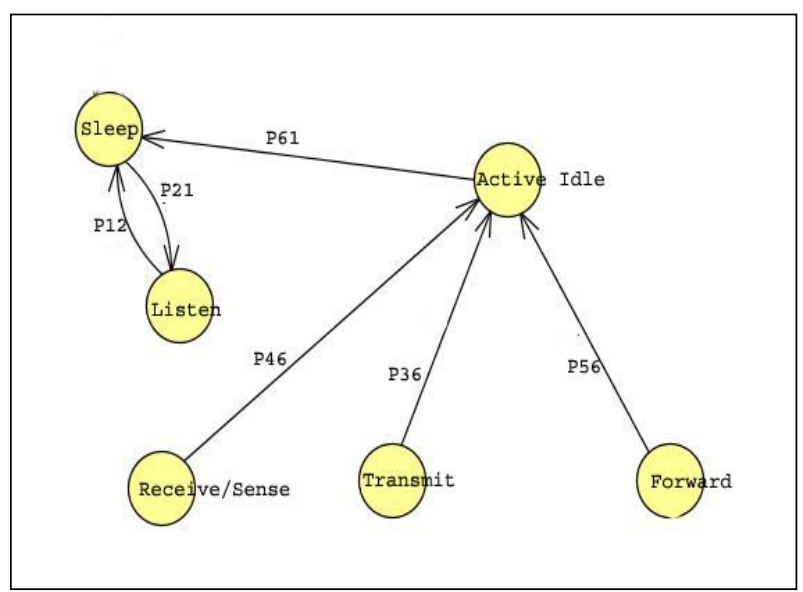

FIG-I NON EVENT condition

In the above transition diagram not much energy is expended from one state to another hence we can assume it to be NONEvent

Now state diagram for EVENT 


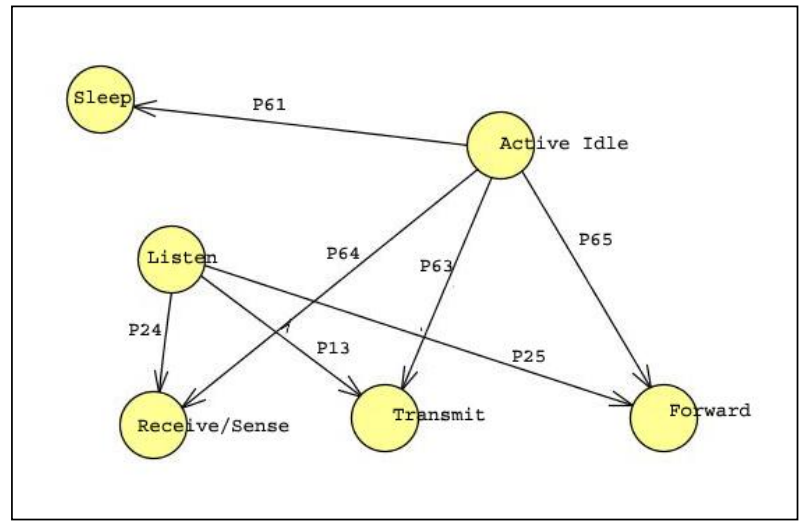

FIG-II EVENT condition

In the above transition diagram appreciable energy is expended from one state to another hence we can assume it to be Event

\section{EVALUATION OF DUTY CYCLE}

We assume that sensors can be considered as a Markov chain, which are locally time synchronized and a feature common time slot length T. Although being common across all sensor nodes the time slot length is not fixed, but is rather computed along with the other input parameters (ie. Working duty cycle and coefficient) based on the model.

A working schedule Ws can be defined as

$\mathrm{Ws}=[\mathrm{s} 0, \ldots, \mathrm{si}, \ldots, \mathrm{sN}-1]$ is an $\mathrm{N} \mathrm{X} 1$ binary vector where

$\mathrm{Si}=\{0$ if the sensor describes NON-Event, 1 if the sensor describes Event

A Markov chain is defined by the transition probabilities $\operatorname{Pr}[\mathrm{si}=1 \mid \mathrm{s}(\mathrm{i}-1)=0]=\alpha$ and $\operatorname{Pr}[\mathrm{si}=0 \mid \mathrm{s}(\mathrm{i}-1)=1]=\beta$ The remaining transition probability are $\operatorname{Pr}[\mathrm{si}=1 \mid \mathrm{s}(\mathrm{i}-1)=0]=1-\beta$

and $\operatorname{Pr}[\mathrm{si}=0 \mid \mathrm{s}(\mathrm{i}-1)=1]=1-\alpha$. Based on the values of $\alpha$ and $\beta$ we obtain the following matrix notation

$\mathrm{P}=[\mathrm{pij}]==\left(\begin{array}{cc}1-\alpha & \alpha \\ \beta & 1-\beta\end{array}\right)$

Where $p i j=\operatorname{Pr}[s i=j \mid s(t-1)=i]$. The probability mass function pmf of the stationary distribution is

$\mathrm{P}=[\mathrm{pi}]=\left(\begin{array}{ll}\mu_{0} & \mu_{1}\end{array}\right)=\left[\begin{array}{ll}\frac{\beta}{\alpha+\beta} & \frac{\alpha}{\alpha+\beta}\end{array}\right]$

where working schedules with memory coefficient is $\gamma=\alpha+\beta$ and working schedule duty cycle is $\mu=\alpha /(\alpha+\beta)$

In WSN scenario the duty cycle is usually an input parameter to the working schedule generator provided by the energy harvesting controller or set prior to the deployment. The values of $\alpha$ and $\beta$ can be computed from the target stationary probability $\mu 1$ and the memory coefficient $\gamma$ as follows

,$\alpha=\gamma \mu 1$

,$\beta=\gamma-\alpha$

$\beta<=1$ and $\alpha>=0$ has to hold, thus memory coefficient $\gamma=[0,1 /(1-\mu 1)]$
Thus the $\gamma$ closed interval is split into 3 regions

Ie. $\gamma=1$ the system is in memory-less state since the transition probabilities are independent of one another

, $\gamma !=1$ the system is not in memory-less condition the likelihood of the transitions between the states also decreases.

When $\gamma$ increases from 1 to $1 /(1-\mu 1)$ the transition between the states become more likely.

Considering the diagram in FIG-I we discuss the non event condition where the following transition probabilities like $\mathrm{P} 12, \mathrm{P} 21, \mathrm{P} 61, \mathrm{P} 46, \mathrm{P} 36, \mathrm{P} 56$ are considered .It is assumed that the remaining transition probabilities do not exert at this moment of non event hence they can be considered as zero.

Hence the expression values of $\pi 1, \pi 2, \pi 3, \pi 4, \pi 5, \pi 6$ has to be redefined so that they can be accommodated for non event case.

$$
\begin{aligned}
& \pi_{1}=\frac{1}{D} \\
& \pi_{2}=\frac{P_{12}}{D} \\
& \pi_{3}=\frac{P_{61} P_{13}+P_{61} P_{23} P_{12}+P_{63}\left(1-P_{12} P_{21}\right)}{P_{61} D}=0 \\
& \pi_{4}=\frac{P_{61} P_{12} P_{24}+P_{64}\left(1-P_{21} P_{12}\right)}{P_{61} D}=0 \\
& \pi_{5}=\frac{P_{61} P_{12} P_{25}+P_{65}\left(1-P_{21} P_{12}\right)}{P_{61} D}=0 \\
& \pi_{6}=\frac{\left(1-P_{21} P_{12}\right)}{P_{61} D} \\
& \text { Where } D=1+P_{12}+\frac{1-P_{21} P_{12}}{P_{61}}
\end{aligned}
$$

Now let us consider E1,E2,E3,E4,E5,E6 the energy values of the states sleep, listen, active-transmit, active-receive, activeforward, and active-idle respectively, the we can find the total energy consumption of the non event condition.

Hence the total energy is given by

ETota 1 NON-EVENT=

$\pi 1 \mathrm{E} 1+\pi 2 \mathrm{E} 2+\pi 3 \mathrm{E} 3+\pi 4 \mathrm{E} 4+\pi 5 \mathrm{E} 5+\pi 6 \mathrm{E} 6=\pi 1 \mathrm{E} 1+\pi 2 \mathrm{E} 2+\pi 6 \mathrm{E} 6$

Similarly we can evaluate for Event condition where the following transition probabilities like P24, P13, P25, P64, P63, P65, and P61 are considered and remaining transition probability is considered as zero as they are non exerting.

Hence we have,

$$
\begin{aligned}
& \pi_{1}=\frac{1}{D} \\
& \pi_{2}=\frac{P_{12}}{D}=0
\end{aligned}
$$




$$
\pi_{3}=\frac{P_{61} P_{13}+P_{61} P_{23} P_{12}+P_{63}\left(1-P_{12} P_{21}\right)}{P_{61} D}=\frac{P_{61} P_{13}+P_{63}}{P_{61} D} \quad \begin{aligned}
& \text { Where }, \\
& \Lambda=\lambda_{t}+\lambda_{r}+\lambda_{f}
\end{aligned}
$$

$$
\pi_{4}=\frac{P_{61} P_{12} P_{24}+P_{64}\left(1-P_{21} P_{12}\right)}{P_{61} D}=\frac{P_{64}}{P_{61} D}
$$$$
\pi_{5}=\frac{P_{61} P_{12} P_{25}+P_{65}\left(1-P_{21} P_{12}\right)}{P_{61} D}=\frac{P_{65}}{P_{61} D}
$$$$
\pi_{6}=\frac{\left(1-P_{21} P_{12}\right)}{P_{61} D}=\frac{1}{P_{61} D}
$$$$
\text { Where } D=1+\mathrm{P}_{13}+\frac{1+\mathrm{P}_{63}+\mathrm{P}_{64}+\mathrm{P}_{65}}{P_{61}}
$$

Now let us consider $\mathrm{E}_{1}, \mathrm{E}_{2}, \mathrm{E}_{3}, \mathrm{E}_{4}, \mathrm{E}_{5}, \mathrm{E}_{6}$ the energy values of the states sleep, listen, active-transmit, active-receive, activeforward, and active-idle respectively, the we can find the total energy consumption of the event condition.

Hence the total energy is given by

$\mathrm{E}_{\text {Tota }} \quad 1 \quad$ EVENT $=\pi_{1} \mathrm{E}_{1}+\pi_{2} \mathrm{E}_{2}+\pi_{3} \mathrm{E}_{3}+\pi_{4} \mathrm{E}_{4}+\pi_{5} \mathrm{E}_{5}+\pi_{6} \mathrm{E}_{6}=\pi_{1} \mathrm{E}_{1}$ $+\pi_{3} \mathrm{E}_{3}+\pi_{4} \mathrm{E}_{4}+\pi_{5} \mathrm{E}_{5}+\pi_{6} \mathrm{E}_{6}$

So the probability due to non Event and Event condition can be obtained as

$$
\begin{gathered}
\alpha=\frac{E_{\text {Tota l NON-EVENT }}}{E_{1}+E_{2}+E_{3}+E_{4}+E_{5}+E_{6}} \ldots \mathrm{I} \\
\beta=\frac{E_{\text {Tota l EVENT }}}{E_{1}+E_{2}+E_{3}+E_{4}+E_{5}+E_{6}} \ldots \text { II }
\end{gathered}
$$

Now we have the effective duty cycle from basic definition that

Duty cycle (DS)

Total Time spent for Event

Total Time spent for Event+ Total time spent for Non-Event

$$
=\frac{\mathrm{T} 13+\mathrm{T} 24+\mathrm{T} 25+\mathrm{T} 61+\mathrm{T} 64+\mathrm{T} 63+\mathrm{T} 65}{(T 13+T 24+T 25+T 61+T 64+T 63+T 65)+}
$$

$(T 61+T 56+T 36+T 46+T 12+T 21)$

$=$

$$
\frac{\left[\frac{1-e^{-\lambda_{t} T_{s}}}{\lambda_{t}}+2\left\{\frac{1-e^{-\left(\Lambda T_{l}\right)}}{\Lambda}\right\}+3\left\{\frac{1-e^{-\left(\Lambda T_{a}\right)}}{\Lambda}\right\}\right]}{\left[\frac{1-e^{-\lambda_{t} T_{s}}}{\lambda_{t}}+2\left\{\frac{1-e^{-\left(\Lambda T_{l}\right)}}{\Lambda}\right\}+3\left\{\frac{1-e^{-\left(\Lambda T_{a}\right)}}{\Lambda}\right\}\right]+\left[\mathrm{Ts}+\frac{1}{\mu_{t}}+\frac{1}{\mu_{r}}+\frac{1}{\mu_{f}}+\mathrm{Tl}+\mathrm{Ts}\right]}
$$

\section{NUMERICAL EXAMPLE}

Based on the on the power consumptions as the ones depicted in tables I and II based on the energy model described in $[2,3,4]$ and the protocol for protocol Basic energy conservation algorithm (BECA) [5] we have,

Table-I Traffic characteristics

\begin{tabular}{|c|c|}
\hline Parameter & Value \\
\hline$T s$ & $10 / 3600 \mathrm{~h}$ \\
\hline$T l$ & $10 / 3600 \mathrm{~h}$ \\
\hline$T a$ & $10 / 3600 \mathrm{~h}$ \\
\hline$\lambda t$ & $3600 / 210 / \mathrm{h}$ \\
\hline$\lambda r$ & $3600 / 21 / \mathrm{h}$ \\
\hline$\lambda f$ & $3600 / 21 / \mathrm{h}$ \\
\hline
\end{tabular}

Table-II Parameters values of power consumption

\begin{tabular}{|c|c|}
\hline Parameter & Value \\
\hline E1 & $0.025 \mathrm{~W}$ \\
\hline$E 2$ & $1.15 \mathrm{~W}$ \\
\hline$E 3$ & $1.6 \mathrm{~W}$ \\
\hline$E 4$ & $1.2 \mathrm{~W}$ \\
\hline$E 5$ & $1.6 \mathrm{~W}$ \\
\hline$E 6$ & $0.13 \mathrm{~W}$ \\
\hline
\end{tabular}

Using the above values we represent the steady state probabilities in a tabular Format for the protocol

\begin{tabular}{|c|c|}
\hline Probability & Value \\
\hline P12 & 0.953 \\
\hline$P 13$ & 0.047 \\
\hline$P 21$ & 0.368 \\
\hline$P 23$ & 0.0301 \\
\hline$P 24$ & 0.0301 \\
\hline$P 25$ & 0.0301 \\
\hline$P 36$ & 1 \\
\hline$P 46$ & 1 \\
\hline
\end{tabular}




\begin{tabular}{|c|c|}
\hline P56 & 1 \\
\hline P61 & 0.368 \\
\hline P63 & 0.0301 \\
\hline$P 64$ & 0.0301 \\
\hline$P 65$ & 0.0301 \\
\hline
\end{tabular}

Assuming the above data and using equation I and II we have, $\alpha=0.0375 ; \beta=0.7125$ then $\mathrm{DS}=0.66$

\section{CONCLUSION}

This analytical study will help to find out the duty cycle for routing protocols, like (BECA) which we have done here. Evaluation of duty cycle is very important to understand the sustainability and energy consumption of any routing protocol under evaluation. This analysis if considered as a tool it will be helpful to determine on the fly duty cycle values. This information can further be utilized to change a given Ws which can be varied in order to improve the performance of the routing protocol under consideration

\section{REFERENCES}

[1] Ghidini ,G and Das, S.K 2012 Energy efficient Markov chain based duty cycling Schemes for greener wireless Sensor networks.ACM J. Emerg. Technol. Comput. Syst. Article 29 (October 2012), 32 pages.
[2] Stemm ,M;Katz,R.H . Measuring and reducing energy consumption of network interfaces in hand held devices.IEICE Trans. Commun.1997, E80-B,1125-1131.

[3] Xu, Y.; Heidemann, J.; Estrin, D. Geography-informed energy conservation for ad hoc routing. In Proceedings of the ACM IGMOBILE Annual International Conference on Mobile Computing and Networking, Rome, Italy, July 16-21, 2001; pp. 70-84.

[4] Chen, B.; Jamieson, K.; Balakrishnan, H.; Morris, R. Span: An energy-efficient coordination algorithm for topology maintenance in ad hoc wireless networks. Wirel. Netw. 2002, 8, 85-96.

[5] Xu, Y.; Heidemann, J.; Estrin, D. Adaptive Energyconservation Routing for Multi-hop Ad Hoc Networks; Technical Report 527; USC/Informaton Sciences Institute: Marina 1 Rey, CA, USA,2000.

[6] Schugers, C.; Tsiatsis, V.; Srivastava, M.B. STEM: topology management for energy efficient sensor networks. In Proceedings of the IEEE Aerospace Conference, Big Sky, MT, USA, March 9-16, 2002.

[7] Xu, Y.; Heidemann, J.; Estrin, D. Geography-informed energy conservation for ad hoc routing. In Proceedings of the ACM SIGMOBILE Annual International Conference on Mobile Computing and Networking, Rome, Italy, July 16-21, 2001; pp. 70-84.

[8] Chung, Y.W.; Hwang, H.Y. Modeling and Analysis of Energy Conservation Scheme Based on Duty Cycling in Wireless Ad Hoc Sensor Network. Sensors 2010, 10, 5569-5589. 